\title{
Analysis of the Promoter Region of the Gene Encoding Sodium/Hydrogen Exchanger 1 Protein
}

\author{
Gharelo RZ* and Bandehagh A
}

Faculty of Agriculture, Department of Plant Breeding and Biotechnology, University of Tabriz, East Azerbaijan, Iran

\begin{abstract}
Understudying gene regulatory network in different plants is essential to engineer plants against biotic/abiotic stress. Here, we analyzed 5'UTR to determine which cis-acting elements present at the promoter of canola NHX1 gene, a salt responsive gene. Arabidopsis NHX1 gene sequence was searched in canola (Brassica napus) genome. The putative sequence of $N H X 1$ gene was extracted and analyzed. Many elements were identified that some of them discussed here.
\end{abstract}

Keywords: Brassica; cis-acting element; Gene regulatory network; Transcription factor

\section{Introduction}

Soil salinization is one of the main growing problems in the agriculture section around the world. Presence of sodium chloride $(\mathrm{NaCl})$ in irrigation water leads to accumulation of ions, such as $\mathrm{Na}^{+}$ and $\mathrm{Cl}^{-}$, in soil. Soils with high level of salinization impair the plant growth by limiting access to water for the plant, osmotic stress. In addition, $\mathrm{Na}^{+}$and $\mathrm{Cl}^{-}$ions taken up in large amount by root interrupts metabolic processes and declines photosynthetic efficiency [1]. In response to such environmental stresses, plants have evolved complex morphological, physiological and biochemical mechanisms that are regulated by gene regulatory networks. Transcription factors are major player in the network controlling a set of gene expression through binding some specific regulatory regions in the genome [2]. One of the main genes are expressed in response to the high level of $\mathrm{Na}^{+}$and $\mathrm{Cl}^{-}$ions in the cell is the $\mathrm{NHX} 1$ gene encoding $\mathrm{Na}^{+}$and $\mathrm{Cl}^{-}$exchanger 1 protein. This membranous protein sequestrates excessive $\mathrm{Na}+$ to vacuole to detoxify the cell from harmful effects [1]. Here, we analyzed 5 ' UTR of canola NHX1 gene through computational based methods. To best our knowledge, there is limit reports about the regulatory regions of NHX1 gene in canola.

\section{Results and Discussion}

In this study, the NHX1 gene from Arabidopsis (id: AT5G27150.1) was blasted against Brassica napus genome (https://www.ncbi.nlm. nih.gov/genome/?term=brassica\%20napus). Canola putative NHX1 gene sequence was extracted from the genome. Using this sequence, the promoter region was predicted by PlantProm (available at http:// mendel.cs.rhul.ac.uk/ and http://www.softberry.com/) and analyzed for presence of cis-acting regulatory elements by PlantCARE (http:// bioinformatics.psb.ugent.be/webtools/plantcare/html/).

Our results are represented in Figure 1. Blast results showed many hints. The first hit with high score was selected and all genomic sequence of NHX1 gene extracted from Brassica napus genome. NHX1 gene located between 2795854 bp and 2799176 bp at chromosome A6 (NC_027765.2).

\section{Conclusions}

The promoter region was predicted and $500 \mathrm{bp}$ upstream from transcription start site scanned. This region of NHX1 gene showed many cis-acting elements. Three of these important cis-acting elements are described below:

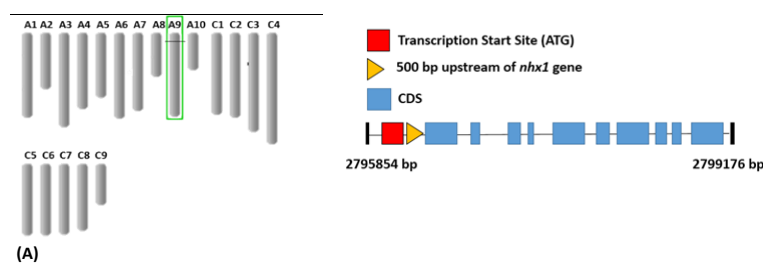

(B)



Figure 1: (A) Location of the NHX1 gene in canola chromosomes, (B) Gene structure of $N H X 1$ gene, (C) Some of important cis-acting element found at 500 bp upstream of canola $N H X 1$ gene.

(a) CAAT box: Common cis-acting elements with sequence of GG(T/C) CAATCT and can be found about 75 bp position regarding to transcription start site, acting as binding site for the CCAAT-binding

*Corresponding author: Dr. Reza Shokri Gharelo, Faculty of Agriculture, Department of Plant Breeding and Biotechnology, University of Tabriz, East Azerbaijan, Iran, Tel: +984133341300; E-mail: shokrireza67@gmail.com

Received November 17, 2017; Accepted November 28, 2017; Published December 01, 2017

Citation: Gharelo RZ, Bandehagh A (2017) Analysis of the Promoter Region of the Gene Encoding Sodium/Hydrogen Exchanger 1 Protein. J Mol Genet Med 11: 312 doi:10.4172/1747-0862.1000312

Copyright: (c) 2017 Gharelo RZ, et al. This is an open-access article distributed under the terms of the Creative Commons Attribution License, which permits unrestricted use, distribution, and reproduction in any medium, provided the original author and source are credited 
factor (CBF), also known as the Nuclear Factor Y (NF-Y) or Hem Activator Protein (HAP). This element has been found at promoter of many genes, including genes regulating flowering in Arabidopsis [3], photosynthetic genes [4], genes encoding heat shock proteins [5] and abiotic stress responsive genes [6].

(b) TC-rich repeats: TC-rich repeat is involved in defense and stress responsiveness. It has found at promoter of osa-MIR396c salt responsive transcript [7], the transcription factor gene TaMYB33 responsive to salt, drought and abscisic acid [8] and copper-containing amine oxidase genes [9].

(c) WUN motifs: The genes harboring this cis-acting element act as wound responsive gene [10]. In addition, studies have demonstrated that WUN-motif is also present at the promoter of the genes responding to drought stress [11], TMV-infection [12], and salinity [13].

\section{References}

1. Deinlein U, Stephan AB, Horie T, Luo W (2014) Plant salt-tolerance mechanisms. Trends Plant Sci 19: 371-379.

2. Nakashima K, Yamaguchi-Shinozaki K, Shinozaki K (2014) The transcriptiona regulatory network in the drought response and its crosstalk in abiotic stress responses including drought, cold, and heat. Front Plant Sci 5: 1-7.

3. Wenkel S, Turck F, Singer K, Gissot L (2006) CONSTANS and the CCAAT box binding complex share a functionally important domain and interact to regulate flowering of Arabidopsis. Plant Cell 18: 2971-2984.

4. Kusnetsov V, Landsberger M, Meurer J, Oelmüller R (1999) The assembly of the CAAT-box binding complex at a photosynthesis gene promoter is regulated by light, cytokinin, and the stage of the plastids. J Biol Chem 274: 36009-36014

5. Chuang $\mathrm{KH}, \mathrm{Ho} \mathrm{SH}$, Song YL (2007) Cloning and expression analysis of heat shock cognate 70 gene promoter in tiger shrimp (Penaeus monodon). Gene 405: 10-18.

6. Khurana N, Chauhan H, Khurana P (2013) Wheat chloroplast targeted sHSP26 promoter confers heat and abiotic stress inducible expression in transgenic Arabidopsis plants. PLoS One 8: 1-14.

7. Gao P, Bai X, Yang L, Lv D (2010) Over-expression of osa-MIR396c decreases salt and alkali stress tolerance. Planta 231: 991-1001.

8. Qin $Y$, Wang M, Tian $Y$, He W (2012) Over-expression of TaMYB33 encoding novel wheat MYB transcription factor increases salt and drought tolerance in Arabidopsis. Mol Biol Rep 39: 7183-7192.

9. Wang W, Wu H, Liu JH (2017) Genome-wide identification and expression profiling of copper-containing amine oxidase genes in sweet orange (Citrus sinensis). Tree Genet Gen 13: 31-41.

10. Ni M, Cui D, Gelvin SB (1996) Sequence-specific interactions of woundinducible nuclear factors with mannopine synthase 2' promoter woundresponsive elements. Plant Mol Biol 30: 77-96.

11. Chen BJ, Wang Y, Hu YL, Wu Q (2005) Cloning and characterization of a drought-inducible MYB gene from Boea crassifolia. Plant Sci 168: 493-500.

12. Hayashi T, Kobayashi D, Kariu T, Tahara M (2003) Genomic cloning of ribonucleases in Nicotiana glutinosa leaves, as induced in response to wounding or to TMV-infection, and characterization of their promoters. Biosci Biotech Biochem 67: 2574-2583.

13. Valifard M, Mohsenzadeh S, Niazi A, Moghadam A (2015) Phenylalanine ammonia lyase isolation and functional analysis of phenylpropanoid pathway under salinity stress in 'Salvia' species. Aust J Crop Sci 9: 656-665. 